\title{
Correspondence
}

\section{A lost cause}

On reading Dr Moorey's earnest response to Dr Gipps' views, I was struck by his description of the 'depressive mode'. ${ }^{\text {. }}$ This marvel of development, 100 years on from Freud's classic paper, ${ }^{2}$ is - in Dr Moorey's view - a 'complex neural network, including multiple relevant brain regions that are activated or deactivated during depression'. This, he argues, is the target of therapeutic practice in cognitive-behavioural therapy (CBT), where unconscious schemas are automatic, not repressed. It seems to the reader that in this dehumanised framework, grief and loss are merely 'problems' that face humankind, which need to be put on the CBT table to be sorted out openly between therapist and patient. The tools? Good old-fashioned common sense, an indefatigably optimistic therapist and wellpositioned intelligence. As for the measures: specially designed scales that measure the very structure which they helped create.

I am writing this piece to explore how both Dr Moorey and Dr Gipps warily circle around a point which is never highlighted in its own right.

Freud's theory of melancholia ${ }^{2}$ posits an unconscious basis for the depressed patient's dilemma. This theory holds true: the proof is in the analytic setting, and all contemporary psychoanalytic approaches which describe the transference find their roots in this classic paper. ${ }^{3}$ Freud writes about an identification of the ego with the abandoned object, saying 'thus the shadow of the object fell upon the ego'. This is still pre-object relations. American psychoanalysis, which was evolving at the time, is well known to have been profoundly influenced by Freud.

In the 1950s, psychoanalysts around the world were working on extending early theories, and the Americans actively participated in this worldwide development. So, with respect, Dr Moorey's point about Beck's 'perfectly acceptable masochism hypothesis' is a bit like Lamarck chasing after amputated tails while Darwin was thinking about evolution!

The crux of the matter is that Beck moved into what is essentially the conscious realm when he developed his theory. Today, anyone who manages patients with depression will know that the latter is the easier and, dare we say, cheaper option. It's all backed up by robust evidence, and supported by what is essentially an Orwellian ${ }^{4}$ environment. I hardly need wonder why patients are rarely asked what they prefer: an analyst who is interested in undiscovered aspects of their loss and is willing to explore themselves in the process, or one bent on pinning down the patient's experience in prosaic terms.

This is why I take exception to the statement in Dr Moorey's final paragraph, which is unreferenced and states that CBT 'has given psychoanalysts methodologies they now use to evaluate their own theories'. This is outrageous, as no self-respecting psychoanalyst would turn to a two-dimensional construct to tell them whether they are tuned into their patient's inner world. I think this is another example of the kind of empiricism that undermines a patient's personal struggle with loss, ignores the depth in a poorly understood psychoanalytic theory and exposes a flawed theoretical argument.
Why would psychoanalysis have any real use for a methodology that doesn't even address its basic theoretical stance? Dr Moorey's thesis is, unfortunately, a lost cause. So, to quote from the excellent choice of title for this debate:

'I give you the mausoleum of all hope and desire [...] I give it to you not that you may remember time, but that you might forget it now and then for a moment and not spend all of your breath trying to conquer it. Because no battle is ever won he said. They are not even fought. The field only reveals to man his own folly and despair, and victory is an illusion of philosophers and fools'. (William Faulkner, The Sound and the Fury ${ }^{5}$ )

Anuradha Menon, Consultant Psychiatrist and Medical Psychotherapist, Leeds and York Partnership NHS Foundation Trust; email: anuradha. menon@nhs.net

1 Moorey S. The cognitive therapy of depression rests on substantial theoretical, empirical and clinical foundations: a reply to Dr Gipps. BJPsych Bull 2017; 41(5): 272-275.

2 Freud S. Mourning and Melancholia. Standard Edition, 14. Hogarth Press, 1917.

3 Steiner J. The conflict between mourning and melancholia. Psychoanal Q 2005; 74(1): 83-104.

4 Orwell G. Animal Farm. Penguin Group, 1946.

5 Faulkner W. The Sound and the Fury. Jonathan Cape \& Harrison Smith, 1929.

doi:10.1192/bjb.2018.1

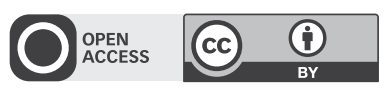

(c) The Author 2018. This is an Open Access article, distributed under the terms of the Creative Commons Attribution licence (http://creativecommons.org/ licenses/by/4.0/), which permits unrestricted re-use, distribution, and reproduction in any medium, provided the original work is properly cited.

\section{Psychiatry, not mental health}

Timms begins his article on solid ground, highlighting the serious problem of psychiatric jargon. ${ }^{1}$ Sadly, it descends into a light-hearted list, focused on the gulf between manager and clinician in the British health service. Most importantly, he omits four gravely misused jargon terms prevalent in psychiatry.

First, the noun 'depression' and its adjective 'depressed'. The noun has half a dozen dictionary definitions unrelated to medicine ${ }^{2}$ and, as a word on its own, it is not a recognised diagnosis in either psychiatric classification. ${ }^{3,4}$ Yet it is used by the lay public, patients and healthcare professionals alike to refer to a medical diagnosis, a single symptom or a normal emotion. It is invariably unclear which meaning is intended by the speaker. This confusion contributes to massive over-prescription of an unfortunately named class of drugs; ${ }^{5}$ anecdotally, it is not uncommon even for senior psychiatrists to justify antidepressant treatment, having cast aside clear 
diagnostic criteria, ${ }^{3,4}$ with comments such as: 'Well, there's definitely a bit of depression there' or 'She says she feels depressed'.

In a similar vein, 'paranoia' and 'paranoid' are often used by clinicians in their lay meaning of 'intense suspicion', ${ }^{2}$ when the true psychiatric definition is 'delusional'; ${ }^{6}$ such ideation might involve purely grandiose or somatic themes. Despite this, one often sees 'paranoid' and 'persecutory' used synonymously. The subjective complaint of 'paranoia' is common in patients with neurotic presentations and personality disorders, and its inappropriate use in case notes without careful use of inverted commas, to signify a verbatim quote, risks inappropriate labelling of patients as psychotic and overtreatment with antipsychotics.

Next, the term 'psychosis' is increasingly used as a diagnosis - as if it were a singular disease for which specific treatments were indicated ${ }^{7}$ - rather than the syndrome that it is. It can occur in organic, substance-induced or affective disorders, yet I contend that 'psychosis' is often used, lazily, as a euphemism for schizophrenia, by psychiatrists either ignorant of established diagnostic criteria ${ }^{3,4}$ or wary of stigmatising their patients (as if one would happily tell guests at a dinner party that one was 'psychotic').

Most concerning, though, is the jargon that Timms includes in his own title: 'mental health'. ${ }^{1}$ The assumption that 'mental health' and the oxymoronic 'mental health disorder' are synonymous with psychiatry and its diseases is quite erroneous. Psychiatry, as practised by psychiatric nurses and psychiatrists, was once charged with the management of patients with psychiatric diseases. But our colleagues are now mental health nurses and our departments mental health services. Far from relating to recognised diseases, the doublespeak 'mental health' has become synonymous with a vague and unattainable concept of complete emotional well-being. Consequently, an increasing fraction of our population, even a majority according to some reports, ${ }^{8}$ young and old, are reported to have 'mental health problems'. The jargon underlying this explosion has set us and our entire healthcare system up to fail, through unrealistic public expectations and ever unmet need.

Let us be psychiatrists and psychiatric nurses once more; let us work in psychiatric services. Let us diagnose schizophrenia and depressive episodes using recognised criteria and be judicious in our use of potentially hazardous and costly treatments; most of all, let us avoid terms steeped in ambiguity.

Richard Braithwaite, Consultant Psychiatrist, Isle of Wight NHS Trust; email: richard.braithwaite@iow.nhs.uk

1 Timms P. A Devil's dictionary for mental health. BJPsych Bull 2017; 41(5): 244-246.

2 Chambers Harrap. The Chambers Dictionary (13th edn). Chambers Harrap Publishers Ltd, 2014.

3 World Health Organization. The ICD-10 Classification of Mental and Behavioural Disorders: Clinical Descriptions and Diagnostic Guidelines. WHO, 1992.

4 American Psychiatric Association. Diagnostic and Statistical Manual of Mental Disorders (5th edn). American Psychiatric Publishing, 2013.

5 Braithwaite R. Evidence suggests massive overdiagnosis and, by extrapolation, overprescription of antidepressants. BMJ 2014; 348: g1436.
6 Hamilton M (ed). Fish's Clinical Psychopathology (2nd edn). Butterworth-Heinemann, 1985.

7 NHS Choices. Psychosis - Treatment. NHS Choices, 2016. Available at http://www.nhs.uk/Conditions/Psychosis/Pages/Treatment.aspx (accessed 3 October 2017)

8 Arie S. Simon Wessely: "Every time we have a mental health awareness week my spirits sink". BMJ 2017; 358: j4305.

doi:10.1192/bjb.2018.2

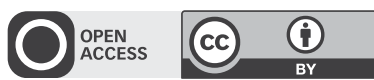

(c) The Author 2018. This is an Open Access article, distributed under the terms of the Creative Commons Attribution licence (http://creativecommons.org/ licenses/by/4.0/), which permits unrestricted re-use, distribution, and reproduction in any medium, provided the original work is properly cited.

\section{Sharing quality and safety improvement work in the field of mental health}

I welcome the findings of D'Lima, Crawford, Darzi and Archer $^{1}$ on the relative scarcity of publications related to mental health quality and safety improvement within reputable quality and safety journals.

I agree with the authors' proposition that there is increasing interest in the application of improvement science within the mental health field. A large number of providers of mental health services in the UK and beyond are now starting to apply quality improvement methods at scale. The Royal College of Psychiatrists has established a quality improvement committee over the past year, and has recently appointed its first quality improvement lead. There is also an organically growing global mental health improvement network (\#MHimprove), which meets twice a year and has begun to present and share knowledge at large international conferences.

As both the College quality improvement lead and the lead for quality at East London NHS Foundation Trust (ELFT), with perhaps one of the largest improvement programmes in the world within mental health services, my experience agrees with the conclusion of the authors that publishing mental health improvement work within reputable quality and safety journals is a struggle. Our efforts to share real-world improvement work have largely been unsuccessful in the high-quality journals within this field. My theory, both as a submitting author and a reader of these journals, is that the journals are still focusing more on the research and evaluation end of the spectrum, as opposed to real-world, messy improvement work in mental health services.

As an example, the use of Shewhart (control) charts to demonstrate improvement over time, which is seen as best practice by improvers across the globe, is still frowned upon by journals (both subject-matter specialist journals and quality and safety journals), who prefer enumerative statistics in the form of pre- and post-comparison of averages. This jars with the real world of applied science, where there is no pre- and post-state, but a gradual and iterative transition, fuelled by multiple tests of change with increasing degree of belief and reliability in the change package.

Despite these challenges, ELFT has published approximately 15 peer-reviewed articles over the past 4 years and has 